\title{
Analytical theory of oxygen transport in the human placenta
}

\author{
A.S. Serov ${ }^{\text {a,* }}$, C.M. Salafia ${ }^{b}$, M. Filoche ${ }^{a}$, D.S. Grebenkov ${ }^{a}$ \\ a Laboratoire de Physique de la Matière Condensée, Ecole Polytechnique, CNRS, 91128 Palaiseau Cedex, France \\ b Placental Analytics LLC, 93 Colonial Avenue, Larchmont, New York 10538, USA
}

\section{H I G H L I G H T S}

- A 2D+1D stream-tube placenta model is used to describe oxygen exchange.

- Diffusion-convection equation is solved analytically to get oxygen exchange rate.

- Two geometrical characteristics are the most relevant: villi radius and density.

- Two independent parameter combinations characterizing oxygen exchange are identified.

- Diagrams and explicit formulas of oxygen exchange efficiency are provided.

\section{A R T I C L E I N F O}

\section{Article history:}

Received 14 October 2014

Accepted 23 December 2014

Available online 8 January 2015

\section{Keywords:}

Diffusion-convection

Stream-tube placenta model

Optimal villi density

Oxygen exchange efficiency

Pathology diagnostics

G R A P H I C A L A B S T R A C T

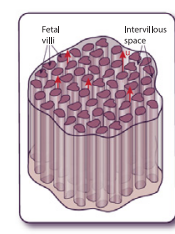

Stream-tube placenta model

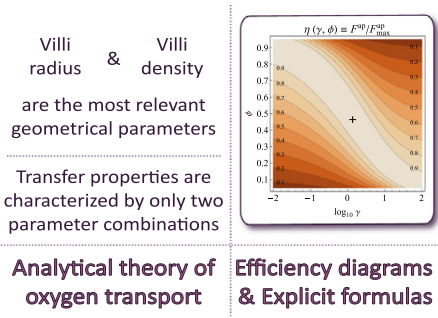

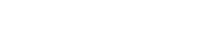

\section{A B S T R A C T}

We propose an analytical approach to solving the diffusion-convection equations governing oxygen transport in the human placenta. We show that only two geometrical characteristics of a placental crosssection, villi density and the effective villi radius, are needed to predict fetal oxygen uptake. We also identify two combinations of physiological parameters that determine oxygen uptake in a given placenta: (i) the maximal oxygen inflow of a placentone if there were no tissue blocking the flow and (ii) the ratio of transit time of maternal blood through the intervillous space to oxygen extraction time. We derive analytical formulas for fast and simple calculation of oxygen uptake and provide two diagrams of efficiency of oxygen transport in an arbitrary placental cross-section. We finally show that artificial perfusion experiments with no-hemoglobin blood tend to give a two-orders-of-magnitude underestimation of the in vivo oxygen uptake and that the optimal geometry for such setup alters significantly. The theory allows one to adjust the results of artificial placenta perfusion experiments to account for oxygen-hemoglobin dissociation. Combined with image analysis techniques, the presented model can give an easy-to-use tool for prediction of the human placenta efficiency.
\end{abstract}

(c) 2014 Elsevier Ltd. All rights reserved.

\section{Introduction}

The human placenta consists of maternal and fetal parts (Fig. 1a). The maternal part is a blood basin which is supplied by spiral arteries and drained by maternal veins (Benirschke et al., 2006). The fetal part is a villous tree, inside which fetal blood goes from umbilical arteries to the umbilical vein through fetal capillaries. Maternal blood percolates

\footnotetext{
* Corresponding author. Tel.: +3316933 47 07; fax: +3316933 4799.

E-mail address: alexander.serov@polytechnique.edu (A.S. Serov).
}

through the same arboreous structure on the outside. Maternal blood and fetal blood do not mix, so the gas and nutrient exchange takes place at the surface of the villous tree, sections of which can be observed in a typical histological 2D placental slide (Fig. 1b). Modeling and understanding the relation between the geometrical structure of the exchange surface of the villous tree and the efficiency of the transport function of the placenta constitutes the central object of our study.

Placenta models have been proposed previously (see discussions in Aifantis, 1978; Battaglia and Meschia, 1986; Chernyavsky et al., 2010; Gill et al., 2011). 1D models dealt with oxygen transport at the 
a

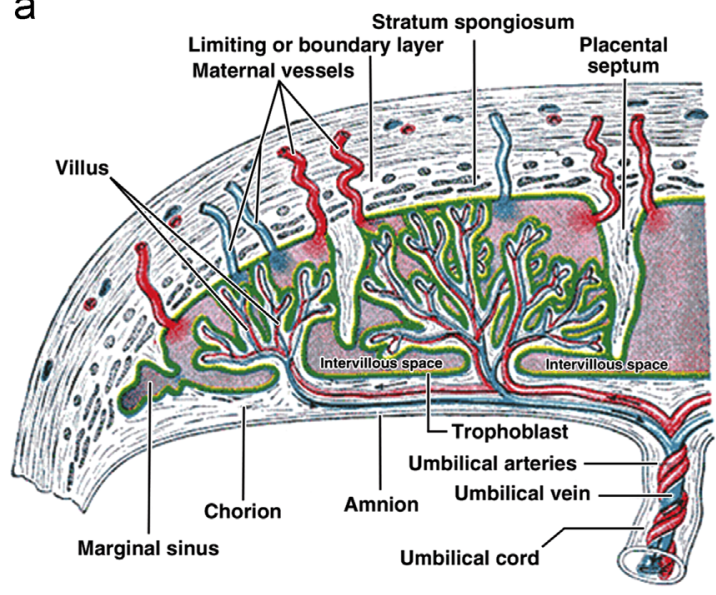

b

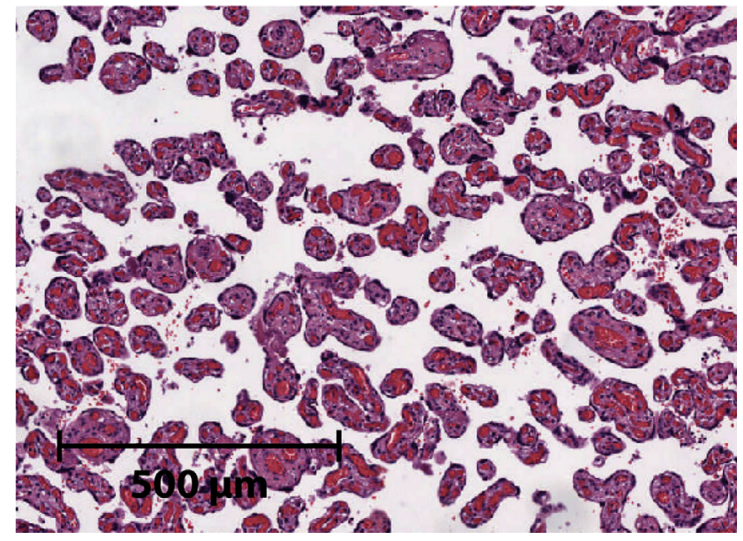

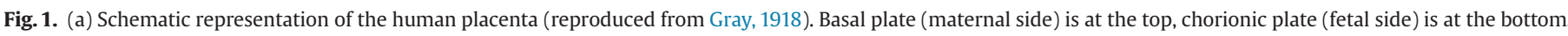

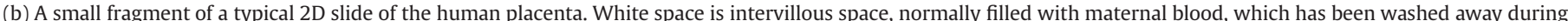

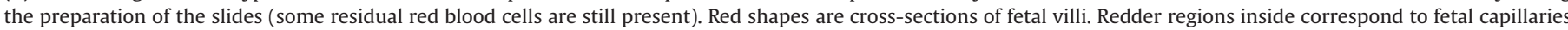

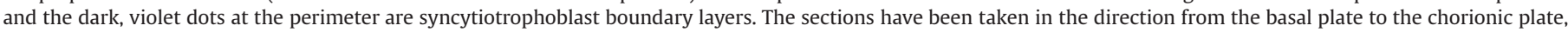
and are H\&E stained. (For interpretation of the references to color in this figure caption, the reader is referred to the web version of this paper.)

scale of either one single villus or the whole placenta, in both cases imposing a flat exchange surface between maternal and fetal blood (Bartels et al., 1962; Shapiro et al., 1967; Kirschbaum and Shapiro, 1969; Hill et al., 1972, 1973; Longo et al., 1972a,b; Power et al., 1972a,b; Lardner, 1975; Wilbur et al., 1978; Groome, 1991); some 2D models were used to study the co-orientation of maternal and fetal flows (Bartels et al., 1962; Metcalfe et al., 1964; Shapiro et al., 1967; Faber, 1969; Kirschbaum and Shapiro, 1969; Guilbeau et al., 1970; Moll, 1972; Schröder, 1982; Battaglia and Meschia, 1986); other models represented the placenta as a porous medium (Erian et al., 1977; Schröder, 1982; Chernyavsky et al., 2010). A lumped element model was also proposed to calculate 1D placental diffusing capacity and to relate morphometric data to the efficiency of gas transport (see Mayhew et al., 1984, 1986 and references therein). To our knowledge, the only 3D placenta model was introduced by Chernyavsky et al. (2010) to study how the position of venous outlets and the existence of a central cavity influences oxygen transport in a hemispherical porous-medium placentone model.

However, none of these models uses fine geometrical structure of experimentally obtained placental slides (Fig. 1b) as direct input. In a recent paper we introduced a stream-tube placenta model (STPM; Fig. 2b), which is built upon histological placental crosssections (Serov et al., 2015) in contrast to previous placenta models. In this model, cross-sections of stream tubes of maternal blood flow (MBF) in the intervillous space of the human placenta were reconstructed from placental cross-sections (Fig. 1b) and virtually extended along the third dimension. Although successive crosssections of a stream tube obviously vary in the placenta, this variation cannot be reproduced from a single cross-section and was ignored in this model. Relevant physiological and geometrical parameters of the model were estimated from the available experimental data. Numerical simulations of oxygen transport for identical circular villi were then performed and showed that the model exhibits an optimal villi density yielding maximal oxygen uptake. Deviations from these optimal characteristics with variations of model parameters were estimated. The obtained optimal villi density $(0.47 \pm 0.06)$ corresponds to that experimentally obtained in healthy human placentas $(0.46 \pm 0.06)$.

The present paper relies on the same STPM, but provides the first approximate analytical theory of oxygen uptake in the human placenta based on histological cross-sections. The present work significantly develops the results of the previous study by:
- allowing for a fast calculation of oxygen uptake for arbitrary placental cross-sections, while only circular villi were considered before;

- demonstrating explicit dependence of oxygen uptake on model parameters and their interrelation, which could not be obtained numerically;

- introducing two uptake efficiency indicators for which analytical formulas and diagrams are provided;

- showing that accounting for oxygen-hemoglobin reaction is important for interpretation of artificial perfusion experiments (with no-Hb blood) and providing a method of recalculation of the results of such experiments to account for oxygen-hemoglobin reaction.

In the following, the construction of the analytical theory is preceded by a full description of the geometrical model, physical assumptions and parameters describing the human placenta.

\section{The model}

\subsection{Model assumptions}

Maternal blood arrives into the intervillous space of the human placenta by spiral arteries (Fig. 2a). It then percolates through the branching structure of a tree of fetal villi and leaves the intervillous space by decidual veins. The total pattern of the MBF can be virtually subdivided into small regions (stream tubes), each following the flow and extending from the central cavity to a decidual vein. Each stream tube comes into contact with numerous fetal villi, at the surface of which mass exchange between maternal and fetal blood takes place.

We model one such stream tube unfolded as a cylinder of an arbitrary cross-section containing multiple parallel cylinders of arbitrary cross-sections and sizes, which represent fetal villi (Fig. 2b). The shapes and locations of the villi can be taken from a histological slide. Since we aim to base the STPM on histological slides (Fig. 1b) which provide only one stream-tube section without any information about the change of this section along the MBF, we further postulate that the same shapes and locations of villi are conserved along the stream tube. This is obviously an oversimplification of the irregular 3D structure of the placenta, but it is the most 
a

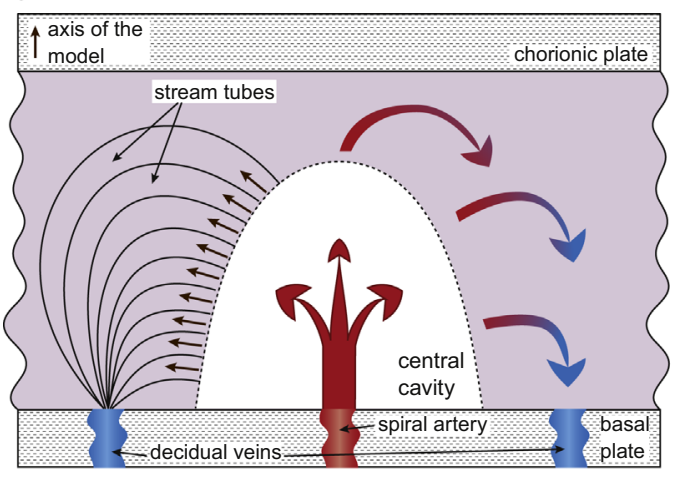

b

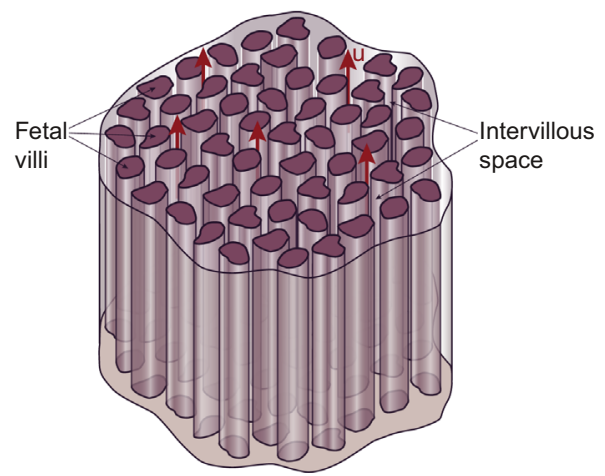

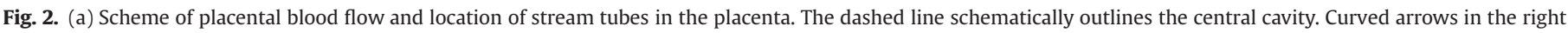

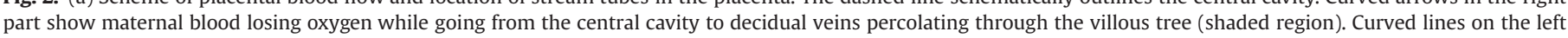

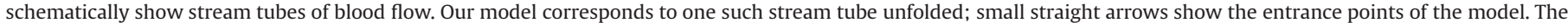

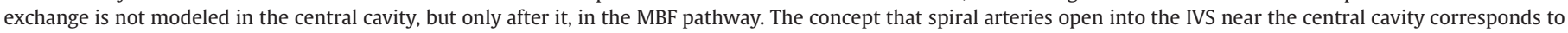

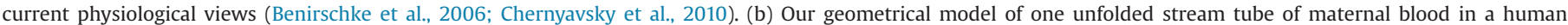

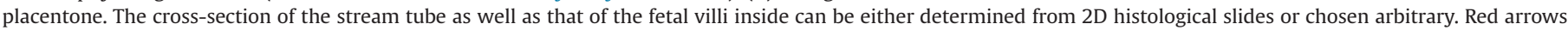
show the flow of maternal blood. (For interpretation of the references to color in this figure caption, the reader is referred to the web version of this paper.)

Table 1

Parameters of the human placenta used in the STPM (see Serov et al., 2015 for the method of calculation of these values).

\begin{tabular}{llc}
\hline Parameter & Symbol & Mean \pm SD \\
\hline Maximal Hb-bound oxygen concentration at $100 \% \mathrm{Hb}$ saturation, $\mathrm{mol} / \mathrm{m}^{3}$ & $c_{\max }$ & $7.30 \pm 0.11$ \\
Oxygen-hemoglobin dissociation constant & $B$ & $94 \pm 2$ \\
Concentration of oxygen dissolved in blood at the entrance to the $\mathrm{IVS}, 10^{-2} \mathrm{~mol} / \mathrm{m}^{3}$ & $c_{0}$ & $6.7 \pm 0.2$ \\
Oxygen diffusivity in blood, $10^{-9} \mathrm{~m}^{2} / \mathrm{s}$ & $D$ & $1.7 \pm 0.5$ \\
Effective villi radius, $10^{-6} \mathrm{~m}$ & $r_{\mathrm{e}}$ & $41 \pm 3$ \\
Permeability of the effective materno-fetal interface, $10^{-4} \mathrm{~m} / \mathrm{s}$ & $w$ & $2.8 \pm 1.1$ \\
Placentone radius, $10^{-2} \mathrm{~m}$ & $R$ & $1.6 \pm 0.4$ \\
Velocity of the maternal blood flow, $10^{-4} \mathrm{~m} / \mathrm{s}$ & $u$ & 6 \\
Stream tube length, $10^{-2} \mathrm{~m}$ & $L$ & 1.6 \\
\hline
\end{tabular}

straightforward assumption given the lack of complete 3D geometrical data spatially resolving all villi.

The model relies on several other assumptions:

1. Fetal blood is considered as a perfect oxygen sink.

2. MBF is considered to be laminar with slip conditions at all boundaries (no liquid-wall friction), so that the velocity profile in any cross-section is flat.

3. The oxygen-hemoglobin dissociation curve is linearized in the physiological range of partial pressure of oxygen $(0-60 \mathrm{mmHg})$ observed in the human placenta.

4. Oxygen uptake occurs at the feto-maternal interface, i.e. at the boundaries of the small cylinders, and is directly proportional to the interface permeability and to oxygen concentration on the maternal side of the interface.

5. In a cross-section perpendicular to the MBF, oxygen is only redistributed by diffusion.

6. Erythrocytes are uniformly distributed in the maternal blood.

7. Oxygen bound to hemoglobin does not diffuse; only oxygen dissolved in the blood plasma does.

8. Oxygen uptake is stationary.

The validity of these assumptions is thoroughly discussed in Serov et al. (2015). The model also includes geometrical and biological parameters, which are listed in Table 1. It will further be shown that fetal oxygen uptake is determined by two parameter combinations which naturally appear in the development of the theory.

\section{Mathematical formulation}

\subsection{Time scales of the system}

We identify three different physical transport processes in the placenta, each of which operates on a characteristic time scale: hydrodynamic blood flow through the IVS characterized by an average velocity $u$ and transit time $\tau_{\text {tr }}$; diffusion of oxygen with characteristic time $\tau_{\mathrm{D}}$; and equilibration between oxygen bound to hemoglobin and oxygen dissolved in the blood plasma with characteristic time $\tau_{\mathrm{p}}$. This last thermodynamic equilibrium is described as equal partial pressure of oxygen in both states. The three times can be estimated as follows:

- $\tau_{\mathrm{tr}}$, the transit time of blood through the IVS, is of the order of $27 \mathrm{~s}$ from the results of angiographic studies at term (Burchell, 1967; Serov et al., 2015).

- $\tau_{\mathrm{D}}$, reflecting oxygen diffusion over a length $\delta$ in the IVS is $\tau_{\mathrm{D}} \sim \delta^{2} / D$, where $D$ is oxygen diffusivity in the blood plasma (Table 1). Either from calculations (Mayhew and Jairam, 2000), or directly from normal placental sections (Fig. 1b), the mean width of an IVS pore can be estimated as $\delta \sim 80 \mu \mathrm{m}$, yielding $\tau_{\mathrm{D}} \sim 4 \mathrm{~s}$.

- $\tau_{\mathrm{p}}$, an equilibration time scale, which includes characteristic diffusion time for oxygen to reach $\mathrm{Hb}$ molecules inside a red blood cell ( $\sim 10 \mathrm{~ms}$, see Foucquier et al., 2013) and typical time of oxygen-hemoglobin dissociation ( $\sim 20 \mathrm{~ms}$ for the slowest 
process, see Yamaguchi et al., 1985). Together these times sum up to $\tau_{\mathrm{p}} \sim 30 \mathrm{~ms}$.

These three characteristic times are related as follows: $\tau_{\mathrm{p}} \ll \tau_{\mathrm{D}} \lesssim \tau_{\text {tr }}$. This relation suggests that oxygen-hemoglobin dissociation can be considered instantaneous as compared to diffusion and convection; the latter two, by contrast, should be treated simultaneously.

\subsection{Equilibrium between bound and dissolved oxygen}

The very fast oxygen-hemoglobin reaction can be accounted for by assuming that the concentration of oxygen dissolved in the blood plasma $\left(c_{\mathrm{pl}}\right)$ and that of oxygen bound to hemoglobin ( $\left.c_{\mathrm{bnd}}\right)$ instantaneously mirror each other's changes. Mathematically both concentrations can be related by equating oxygen partial pressures in these two forms.

Oxygen dissolved in plasma. Because of the low solubility of oxygen in blood, the partial pressure of the dissolved oxygen $\left(p_{\mathrm{O}_{2}}\right)$ can be related to its concentration $\left(c_{\mathrm{pl}}\right)$ using Henry's law:

$p_{\mathrm{O}_{2}}=\frac{k_{\mathrm{hn}}}{\rho_{\mathrm{bl}}} \cdot c_{\mathrm{pl}}$,

where $\rho_{\mathrm{bl}} \approx 1000 \mathrm{~kg} / \mathrm{m}^{3}$ is the density of blood and the coefficient $k_{\mathrm{hn}}$ can be estimated from the fact that a concentration $c_{\mathrm{pl}} \approx 0.13 \mathrm{~mol} / \mathrm{m}^{3}$ of the dissolved oxygen corresponds to oxygen content of $3 \mathrm{ml} \mathrm{O}_{2} / \mathrm{l}$ blood or partial pressure of $13 \mathrm{kPa}$ at normal conditions (Law and Bukwirwa, 1999), yielding $k_{\mathrm{hn}} \sim 7.5 \times 10^{5} \mathrm{mmHg} \mathrm{kg} / \mathrm{mol}$ for the oxygen dissolved in blood.

Hemoglobin-bound oxygen. The partial pressure of the hemoglobin-bound oxygen depends on its concentration through the Hill equation:

$c_{\mathrm{bnd}}=c_{\max } S\left(p_{\mathrm{O}_{2}}\right), \quad S\left(p_{\mathrm{O}_{2}}\right) \equiv \frac{\left(k_{\mathrm{hl}} p_{\mathrm{O}_{2}}\right)^{\alpha}}{1+\left(k_{\mathrm{hl}} p_{\mathrm{O}_{2}}\right)^{\alpha}}$,

where $c_{\max }$ is the oxygen content of maternal blood at full saturation; $k_{\mathrm{hl}} \approx 0.04 \mathrm{mmHg}^{-1}$ and $\alpha \approx 2.65$ are coefficients of the Hill equation, obtained by fitting the experimental curve of Severinghaus (1979, see Fig. 3).

Equilibrium relation between $c_{\mathrm{pl}}$ and $c_{\mathrm{bnd}}$ can then be obtained by substituting Eq. (1) into Eq. (2):

$c_{\mathrm{bnd}}=c_{\max } S\left(\frac{k_{\mathrm{hn}}}{\rho_{\mathrm{bl}}} c_{\mathrm{pl}}\right)$.

\subsection{Diffusive-convective transport of oxygen}

Diffusive-convective transport of oxygen is governed by the mass conservation law for the total concentration of oxygen in a volume of blood:

$\frac{\partial\left(c_{\mathrm{pl}}+c_{\mathrm{bnd}}\right)}{\partial t}+\operatorname{div} \vec{j}_{c_{\mathrm{tot}}}=0$,

where $\vec{j}_{c_{\mathrm{tot}}}$ is the total flux of oxygen, transported both by diffusion and convection for the dissolved form and only by convection (RBCs being too large objects) for the bound form:

$\vec{j}_{c_{\mathrm{tot}}}=-D \vec{\nabla} c_{\mathrm{pl}}+\vec{u}\left(c_{\mathrm{pl}}+c_{\mathrm{bnd}}\right)$,

where $\vec{u}$ denotes the velocity of the MBF and

$\vec{\nabla} \equiv\left\{\frac{\partial}{\partial x} ; \frac{\partial}{\partial y} ; \frac{\partial}{\partial z}\right\}$

Omitting the time derivative in the stationary regime, substituting Eq. (5) into Eq. (4) and choosing $z$ as the direction of the MBF, we obtain

$\Delta c_{\mathrm{pl}}=\frac{u}{D} \frac{\partial\left(c_{\mathrm{pl}}+c_{\mathrm{bnd}}\right)}{\partial z}$,

where $\Delta \equiv \frac{\partial^{2}}{\partial x^{2}}+\frac{\partial^{2}}{\partial y^{2}}+\frac{\partial^{2}}{\partial z^{2}}$ is the Laplace operator. Using the relation (3) between the dissolved and bound oxygen concentrations we then derive an equation for the unknown $c_{\mathrm{pl}}$ only:

$$
\begin{aligned}
\Delta c_{\mathrm{pl}} & =\frac{u}{D} \frac{\partial}{\partial z}\left(c_{\mathrm{pl}}+c_{\mathrm{max}} S\left(\frac{k_{\mathrm{hn}}}{\rho_{\mathrm{bl}}} c_{\mathrm{pl}}\right)\right) \\
= & \frac{u}{D}\left(1+\frac{c_{\mathrm{max}} k_{\mathrm{hn}}}{\rho_{\mathrm{bl}}} S^{\prime}\left(\frac{k_{\mathrm{hn}}}{\rho_{\mathrm{bl}}} c_{\mathrm{pl}}\right)\right) \frac{\partial c_{\mathrm{pl}}}{\partial z} .
\end{aligned}
$$

This equation is non-linear as $c_{\mathrm{pl}}$ appears also in the argument of the derivative of the Hill saturation function $S^{\prime}$. In a first approximation, $S$ can be linearized by assuming $S^{\prime}$ to be constant in the range of partial pressures of oxygen encountered in the human placenta.

\subsection{Linearization of the Hill equation}

The idea of linearization is simple: to replace the sigmoid saturation function (2) with a linear function of $p_{\mathrm{O}_{2}}$. Although it is natural to make the line pass through the origin, the slope of the line may be chosen differently depending on the range of partial pressures in which we approximate the curve (Fig. 3). Data found in the literature indicate that maternal blood in the IVS of the human placenta has $p_{\mathrm{O}_{2}}$ of about $60 \mathrm{mmHg}$ (Rodesch et al., 1992; Jauniaux et al., 2000; Challier and Uzan, 2003). We further suppose that this partial pressure is the maximal value in the IVS and hence delimits the range of the needed linear approximation. Fitting the experimental curve of Severinghaus (1979) in the region $0-60 \mathrm{mmHg}$ with a straight line passing through zero we obtain a linear approximation

$S\left(p_{\mathrm{O}_{2}}\right) \approx \beta_{60} p_{\mathrm{O}_{2}}, \quad S^{\prime}\left(p_{\mathrm{O}_{2}}\right)=\beta_{60} \approx 0.017 \mathrm{mmHg}^{-1}$,

displayed in Fig. 3.

This approximation leads to the following relation between $c_{\text {tot }}$, $c_{\mathrm{pl}}$ and $c_{\mathrm{bnd}}: c_{\mathrm{tot}} \equiv c_{\mathrm{pl}}+c_{\mathrm{bnd}}=c_{\mathrm{pl}} B$, or

$c_{\mathrm{pl}}=\frac{c_{\mathrm{bnd}}}{B-1}=\frac{c_{\mathrm{max}}}{B-1} S\left(p_{\mathrm{O}_{2}}\right), \quad$ where $B \equiv 1+\frac{c_{\mathrm{max}} \beta_{60} k_{\mathrm{hn}}}{\rho_{\mathrm{bl}}}$.

We emphasize here that ignoring oxygen-hemoglobin interaction would be equivalent to setting $B=1$, which would lead to a hundred-fold underestimation of this constant (Table 1).

From Eq. (5), a linearized version of the corresponding total oxygen flux is then

$\vec{j}_{c_{\mathrm{tot}}}=-D \vec{\nabla} c_{\mathrm{pl}}+\vec{u} c_{\mathrm{pl}} B$

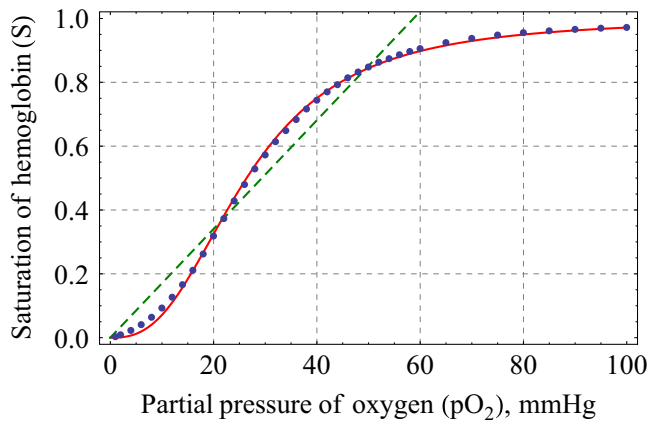

Fig. 3. Oxygen-hemoglobin dissociation curve. In the figure: (i) dots are experimental data at normal conditions as obtained by Severinghaus (1979); (ii) solid curve shows a fit of these data with the Hill equation, the coefficients being $\alpha=2.65, k_{\mathrm{hl}}=0.04 \mathrm{mmHg}^{-1}$; and (iii) straight dashed line is a linear approximation of the curve in the $0-60 \mathrm{mmHg}$ region as discussed in Section 3.4, slope of the line being $\beta_{60} \approx 0.017 \mathrm{mmHg}^{-1}$. 
Finally the partial differential equation (6) becomes

$\Delta c_{\mathrm{pl}}=\frac{u B}{D} \frac{\partial c_{\mathrm{pl}}}{\partial z}$

\subsection{Boundary conditions}

Boundary conditions should be imposed on Eq. (8):

- the boundary of the large cylinder represents the outer boundary of a stream tube. Assuming that there is no exchange of oxygen between different stream tubes, we consider zero flux on its wall:

$$
\frac{\partial c_{\mathrm{pl}}}{\partial n}=0
$$

where $\partial / \partial n$ is the normal derivative directed outside the IVS;

- uptake at the effective feto-maternal interface is proportional to the concentration of oxygen dissolved in the maternal blood plasma:

$D \frac{\partial c_{\mathrm{pl}}}{\partial n}+w c_{\mathrm{pl}}=0$

where $w$ is the permeability of the interface which accounts for the resistance of IVS-villus and villus-capillary membranes as well as for the diffusion in the connective tissue separating the two membranes;

- the total concentration of oxygen in blood is uniform and constant at the entrance of the stream tube $(z=0)$ :

$c_{\mathrm{pl}}(x, y, z=0)=c_{0}, \quad \forall(x, y) \in S_{\mathrm{IVS}}$,

where $c_{0}$ is oxygen concentration in the incoming blood plasma and $S_{\text {IVS }}$ is the part of the stream-tube cross-section occupied by the IVS.

\subsection{Conversion to a $2 D$ eigenvalue equation}

To solve Eq. (8), we separate the coordinate $z$ along the streamtube axis from the coordinates $x$ and $y$ in the transverse crosssection. The general solution of Eq. (8) then takes the following form:

$c_{\mathrm{pl}}(x, y, z)=c_{0} \sum_{j=1}^{\infty} a_{j} v_{j}(x, y) \mathrm{e}^{-\mu_{j} z}$,

where $\left\{\mu_{j}\right\}$ are decay rates in the $z$-direction and $\left\{a_{j}\right\}$ are weights of $c_{\mathrm{pl}}$ in the orthonormal eigenbasis $\left\{v_{j}(x, y)\right\}$ of the Laplace operator $\Delta_{x y}$ in the transverse cross-section. $\left\{v_{j}(x, y)\right\}$ satisfy the following equations:

$$
\begin{cases}\left(\Delta_{x y}+\Lambda_{j}\right) v_{j}=0, & \text { on the stream - tube boundary, } \\ \frac{\partial v_{j}}{\partial n}=0 & \text { on villi boundaries, } \\ \left(\frac{\partial}{\partial n}+\frac{w}{D}\right) v_{j}=0 & \text { in the } z=0 \text { plane } \\ \sum_{j} a_{j} v_{j}=1 & \end{cases}
$$

where

$\Delta_{x y} \equiv \frac{\partial^{2}}{\partial x^{2}}+\frac{\partial^{2}}{\partial y^{2}}, \quad \Lambda_{j} \equiv \mu_{j}^{2}+\mu_{j} \frac{u B}{D}$.

Eigenvalues $\left\{\mu_{j}\right\}$, eigenfunctions $\left\{v_{j}(x, y)\right\}$ and weights $\left\{a_{j}\right\}$ are determined by Eqs. (10)-(14) for a given cross-section. In particular, from Eq. (13) it follows that $a_{j} \equiv \int_{S_{\mathrm{ISS}}} v_{j} d S$, when eigenfunctions are $L_{2^{-}}$ normalized $\left(\int_{S_{\mathrm{VVS}}} v_{j}^{2}=1\right)$.

\subsection{General expression for oxygen uptake}

According to the mass conservation law, oxygen uptake up to length $L$ is equal to the difference between oxygen flow coming into the system at $z=0$ and oxygen flow leaving the system at $z=L$. Using Eqs. (7) and (9) one can derive an explicit dependence of oxygen uptake on the stream-tube length:

$$
\begin{aligned}
F(L) & =\left.\int_{S_{\mathrm{IVS}}}\left(\vec{j}_{c_{\mathrm{tot}}} \cdot \vec{n}_{z}\right)\right|_{z=0} d S-\left.\int_{S_{\mathrm{IVS}}}\left(\vec{j}_{c_{\mathrm{tot}}} \cdot \vec{n}_{z}\right)\right|_{z=L} d S \\
& =c_{0} \sum_{j=1}^{\infty} a_{j}^{2}\left(D \mu_{j}+u B\right)\left(1-\mathrm{e}^{-\mu_{j} L}\right),
\end{aligned}
$$

where the definition of $\left\{a_{j}\right\}$ has been used. This is an exact expression for oxygen uptake, into which the geometrical structure of the placental cross-section enters through the spectral characteristics $\left\{\mu_{j}\right\}$ and $\left\{a_{j}\right\}$. Our goal now is to simplify this expression and to identify the most relevant geometrical and physiological parameters that determine oxygen uptake.

\section{Approximate analytical solution}

\subsection{Form of the approximation}

A quick analysis of Eq. (15) shows that $F(L)$ is a smooth monotonous curve which is linear at small lengths and exponentially saturates at large lengths. In a first approximation, Eq. (15) can then be replaced by an expression which has the same behavior at these limits:

$F^{\mathrm{ap}}(L)=A\left(1-\mathrm{e}^{-\alpha L}\right)$,

where $A$ and $\alpha$ are two parameters: $A$ is oxygen uptake at $L \rightarrow \infty$ (equal to the total incoming oxygen flow), and $\alpha$ is the mean decay rate of oxygen concentration with stream-tube length. We will now relate $A$ and $\alpha$ to the parameters of the model.

\subsection{Uptake at the infinite length}

Large lengths are characterized by saturation when all incoming oxygen is transferred to the fetal blood.

An exact expression for the saturation limit can be obtained from Eq. (15) as $L \rightarrow \infty$ :

$F_{\infty}=c_{0} u B S_{\text {IVS }}+c_{0} D \sum_{j=1}^{\infty} a_{j}^{2} \mu_{j}$

where the identity $\sum_{j=1}^{\infty} a_{j}^{2}=S_{\text {IVs, }}$ resulting from Eq. (13), was used.

Note that the flow in Eq. (17) includes two contributions: convective flow (the first term) and diffusive flow along the $z$-axis (the second term). It turns out that the second term is much smaller than the first one, so that Eq. (17) can be simplified by omitting the diffusive term:

$F_{\infty} \approx A=c_{0} u B S_{\text {IVS }}$.

This approximation is justified by the following arguments:

1. Relative roles of convection and diffusion in a hydrodynamic problem are described by the Péclet number, which is the ratio of characteristic times of diffusive and convective transport to the same distance $\delta: P e=u \delta / D$. Large Péclet numbers ( $P e \gg 1$ ) indicate predominant convective transport, whilst small values signify prevalence of the diffusive transport.

For the human placenta, the ratio $u / D$ is of the order of $10^{5} \mathrm{~m}^{-1}$ (see Table 1), which yields that $P e \gg 1$ for lengths $\delta \gg 10 \mu \mathrm{m}$. As the characteristic length of the stream tube is $L_{0} \sim 1.6 \mathrm{~cm} \gg 10 \mu \mathrm{m}$, we conclude that diffusion along the 
stream tube can be omitted as compared to convection. At the same time, diffusion in the cross-section cannot be ignored as it is the only in-plane mechanism of oxygen transport.

2. Since $99 \%$ of oxygen is bound to hemoglobin in red blood cells (RBC), and RBCs are too large to diffuse, the error from ignoring the diffusive transport term in $F_{\infty}$ does not exceed $1 \%$ in terms of oxygen content. as

Mathematically, the simplification we have used can be written

$\mu_{j} \ll u B / D$,

so that Eq. (14) becomes $\mu_{j} \approx \Lambda_{j} D /(u B)$. It should be noted that statement (19) does not contradict with the fact that $\left\{\mu_{j}\right\}$ grow to infinity with eigenvalue number $j$. In fact, each $\mu_{\mathrm{j}}$ contributes to the final expression with a weight $a_{j}$, which diminishes with $j$. Eq. (19) should be then understood as only valid for all eigenvalues that have significant contributions $a_{j}$.

Oxygen uptake (15) can then be approximated as

$F(L) \approx c_{0} u B S_{\text {IVS }}\left(1-\sum_{j=1}^{\infty} \frac{a_{j}^{2}}{S_{\text {IVS }}} \exp \left(-\frac{D}{u B} \Lambda_{j} L\right)\right)$.

\subsection{Average concentration decay rate}

Using Eq. (18) and comparing Eq. (20) with the approximate form of oxygen uptake (16), one obtains the following definition of the average concentration decay rate $\alpha$ :

$\alpha(L) \equiv-\frac{1}{L} \ln \left(\sum_{j=1}^{\infty} \frac{a_{j}^{2}}{S_{\mathrm{IVS}}} \exp \left(-\frac{D}{u B} \Lambda_{j} L\right)\right)$

In this formula, $\alpha$ depends explicitly on $L$ and implicitly on the cross-sectional geometry. Our goal now is to extract the main part of this implicit dependence. For this purpose, we integrate Eq. (10) over the IVS in the cross-section $\left(S_{\text {IVS }}\right)$. We further apply the divergence theorem (Arfken et al., 2005) to transform the integral over the IVS to an integral over its boundary $\left(P_{\text {tot }}\right.$, which includes the perimeter $P$ of the absorbing boundary of the villi and that of the outer boundary of the stream tube in a cross-section):

$\Lambda_{j}=-\frac{\int_{P_{\mathrm{tot}}} \partial v_{j} / \partial n d P}{\int_{S_{\mathrm{IVS}}} v_{j} d S}=\frac{w}{D} \frac{\int_{P} v_{j} d P}{\int_{S_{\mathrm{IVS}}} v_{j} d S}=\left(\frac{w P}{D S_{\mathrm{IVS}}}\right) q_{j}^{2}$,

where boundary conditions (11) and (12) were used to remove the contribution from the non-absorbing boundary and

$q_{j}^{2} \equiv \frac{\frac{1}{P} \int_{P} v_{j} d P}{\frac{1}{S_{\mathrm{IVS}}} \int_{\mathrm{S}_{\mathrm{IVS}}} v_{j} d S}$ is the dimensionless ratio of the mean value of the eigenfunction $v_{j}$ over the villi boundary to its mean value in the IVS.

In the first approximation, the coefficient $w P /\left(D S_{\text {IVS }}\right)$ in Eq. (22) describes the dependence of $\Lambda_{j}$ on the cross-sectional geometry. Introducing $q_{j}$ and the dimensionless length $\ell(L) \equiv \mathrm{wPL} /\left(\mathrm{uBS}_{\mathrm{IVS}}\right)$ into Eq. (21), we transform it into

$\alpha=\frac{w P}{u B S_{\mathrm{IVS}}} \kappa(L), \quad$ where $\kappa(L) \equiv-\frac{1}{\ell(L)} \ln \left(\sum_{j=1}^{\infty} \frac{a_{j}^{2}}{S_{\mathrm{IVS}}} \exp \left(-\ell(L) q_{j}^{2}\right)\right)$

is a dimensionless coefficient depending on $L$ and the crosssectional geometry and containing fine details of a given villi distribution and shapes, which are ignored by the integral parameters $P$ and $S_{\text {IVs. }}$. Fig. 4a shows the dependence of $\kappa(L)$ on villi density $\left(\phi \equiv 1-S_{\text {IVS }} / S_{\text {tot }}=S_{\text {vil }} / S_{\text {tot }}\right.$, the part of the cross-section occupied by fetal villi) as calculated numerically for a stream-tube of circular cross-section filled with circular villi. One can see that in a first approximation, $\kappa(L)$ can be considered as independent of the cross-sectional geometry in a wide range of biologically relevant stream-tube lengths. For each $L$, the value of $\kappa$ can be determined from Fig. 4b. For the average stream-tube length $\left(L_{0}=1.6 \mathrm{~cm}\right.$, Table 1$), \kappa \approx 0.35$. Using Eqs. (18) and (23), the approximate oxygen uptake (16) can then be rewritten as

$F^{\mathrm{ap}}(L)=c_{0} \mathrm{uBS}_{\mathrm{IVS}}\left(1-\exp \left(-\frac{\mathrm{wPL}}{\mathrm{uBS}_{\mathrm{IVS}}} \kappa(L)\right)\right)$.

\subsection{Dimensionless geometrical parameters}

In Eq. (24), both geometrical parameters $P$ and $S_{\text {IVs }}$ depend on the size of the analyzed region. To facilitate physical analysis of the approximate solution and its comparison to experimental data, we identify two geometrical characteristics of a placental cross-section that are independent of the size of the region:

- The fraction of the cross-section occupied by fetal villi, which we define as the ratio of the total area of villi in a cross-section to the total area of the cross-section: $\phi \equiv S_{\mathrm{vil}} / S_{\text {tot }}$.

- The effective villi radius, which we define as $r_{\mathrm{e}} \equiv 2 S_{\mathrm{vil}} / P \equiv 2 \phi S_{\mathrm{IVS}} /(P(1-\phi))$. In morphometric studies, the inverse parameter $2 / r_{\mathrm{e}}=P / S_{\text {vil }}$ is known as "villi surface density". For circular villi of radius $r, r_{\mathrm{e}} \equiv r$. The mean value of $r_{\mathrm{e}}$ for the human placenta can be found in Table 1 .

Substitution of these definitions into Eq. (24) gives

$\zeta(\gamma, \phi) \equiv \frac{F^{\mathrm{ap}}(\gamma, \phi)}{F_{0}}=(1-\phi)\left(1-\exp \left(-\gamma\left(r_{\mathrm{e}}, L\right) \frac{\phi}{1-\phi}\right)\right)$, a

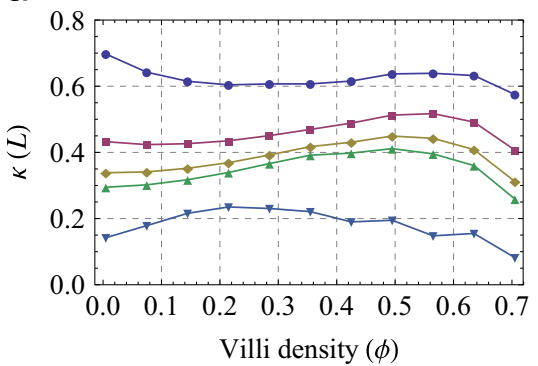

b

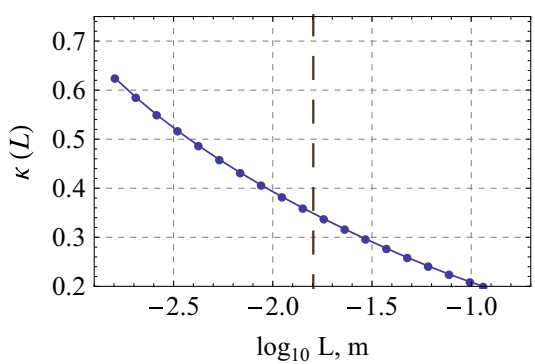

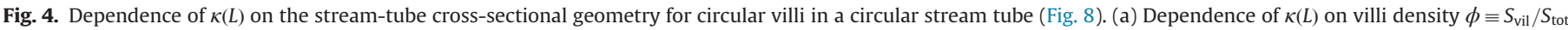

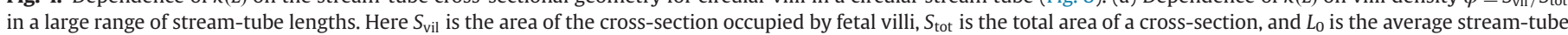

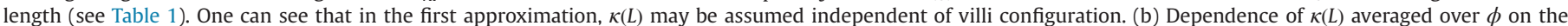
stream-tube length $L$. Dashed vertical line denotes the average stream-tube length $L_{0}$. 
where

$\gamma\left(r_{\mathrm{e}}, L\right) \equiv \frac{2 w \kappa}{u B r_{\mathrm{e}}} L, \quad F_{0} \equiv c_{0} u B S_{\text {tot }}$.

The physical meaning of $F_{0}$ follows from its definition: it is the maximal oxygen flow entering a stream tube that would be achieved if no villi obstructed the IVS of the stream-tube. The incoming flow in the presence of villi is $F_{\infty} \equiv F_{0}(1-\phi)$. Note that $\gamma$ includes information on the average villus shape through the parameter $r_{\mathrm{e}}$, and that $F_{0}$ is independent of the cross-sectional geometry. Normalized oxygen uptake $\zeta(\gamma, \phi) \equiv F^{\text {ap }} / F_{0}$ (which we will call oxygen extraction efficiency) is plotted in Fig. 5a. The physical meaning of $\gamma$ is discussed later.

\subsection{Optimal cross-sectional geometry}

Looking at Fig. 5a and expression (25) for oxygen uptake, it is natural to ask what are the "optimal" values of the parameters $\phi$ and $\gamma$ that maximize oxygen uptake at a given stream-tube length. Fig. 5a clearly shows two trends:

- For any fixed value of $\phi$, larger $\gamma$ provides larger uptake.

- For any fixed value of $\gamma$, there exists some intermediate value of $\phi$ : $0<\phi_{\text {opt }}(\gamma)<1$ that maximizes oxygen uptake. This optimal value $\phi_{\mathrm{opt}}(\gamma)$ tends to diminish with $\gamma$.

Note that from definition (26) it follows that in terms of crosssectional geometrical parameters, an increase of $\gamma$ corresponds to a decrease of the effective radius $r_{\mathrm{e}}$. Fig. $5 \mathrm{a}$ can then be interpreted in the following way: it is more efficient to have many small villi than fewer big villi occupying the same area. This prediction can be understood if one considers the fact that small villi have more absorbing surface per unit of cross-sectional area.

However, in the human placenta, $r_{\mathrm{e}}$ cannot be infinitely small (and hence $\gamma$ infinitely large). Indeed, villi possess an internal structure (e.g., fetal blood vessels) to transport the absorbed oxygen to the fetus. The decrease of $r_{\mathrm{e}}$ below some value is likely making the villi less efficient in transporting the already absorbed oxygen. This argument is supported by an experimental observation that in the terminal and mature intermediate villi of the human placenta (the smallest villi), blood vessels normally occupy the main part of the internal volume. The mean radius of these smallest villi is $r \approx 25-30 \mu \mathrm{m}$ (see Table 28.7 in Benirschke et al., 2006) and is not reported to significantly vary within the same placenta or between different placentas (Benirschke et al., 2006). At the same time, villi density may exhibit significant spatial fluctuations within the same placenta as well as between different placentas (Bacon et al., 1986).

It is then reasonable to reformulate the initial question of optimization of the cross-sectional geometry as which villi density provides the highest oxygen uptake for a given effective villi radius $r_{\mathrm{e}}$. Mathematically, it is the question of finding the maximum of $F$ against $\phi$ for a fixed $\gamma\left(r_{\mathrm{e}}\right)$.

\subsection{Optimal villi density}

Under the constraint of fixed $\gamma$, Eq. (25) implies the existence of maximal uptake at a certain villi density. The reasoning is the following:

- $F(\phi=0)=0$, because the condition $\phi=0$ means no fetomaternal interface and hence no uptake.

- $F(\phi=1)=0$, because fetal vessels occupy the entire crosssection of the stream tube and the incoming MBF is zero as it does not have space to flow.

- $F>0$ for $0<\phi<1$, which corresponds to the fact that the placenta transfers oxygen from mother to fetus for intermediate villi densities. Hence, there always exists a maximal oxygen uptake $F_{\text {max }}(L)$ at a certain villi density $0<\phi_{\text {opt }}(L)<1$ for any $\gamma\left(r_{\mathrm{e}}\right)$.

Here we have used $F$ and not $F^{\text {ap }}$ symbol for oxygen uptake to underline that these arguments are general and are valid not only for the approximate flow, but for the exact flow as well.

The optimal villi density can then be obtained by solving the equation

$$
\begin{aligned}
& \left.\frac{\partial F^{\mathrm{ap}}(\phi, L)}{\partial \phi}\right|_{\phi=\phi_{\mathrm{opt}}}=0 \\
& \text { or } \exp \left(\gamma \frac{\phi_{\mathrm{opt}}}{1-\phi_{\mathrm{opt}}}\right)=1+\frac{\gamma}{1-\phi_{\mathrm{opt}}} .
\end{aligned}
$$

One can note that Eq. (27) is an explicit equation for $\phi_{\text {opt }}$ as a function of $\gamma$ only. As a consequence, it does not require any eigenvalues calculation.

The substitution $x \equiv \gamma \phi_{\text {opt }} /\left(1-\phi_{\text {opt }}\right)$ reduces Eq. (27) to the form $\gamma=g(x)$, where $g(x) \equiv \mathrm{e}^{x}-x-1$, and its solution can be represented as $x=g^{-1}(\gamma)$. Although the inverse function $g^{-1}(\gamma)$ does not have an explicit representation, its form can be easily calculated once and then the tabulated values can be used in practice. Returning to the
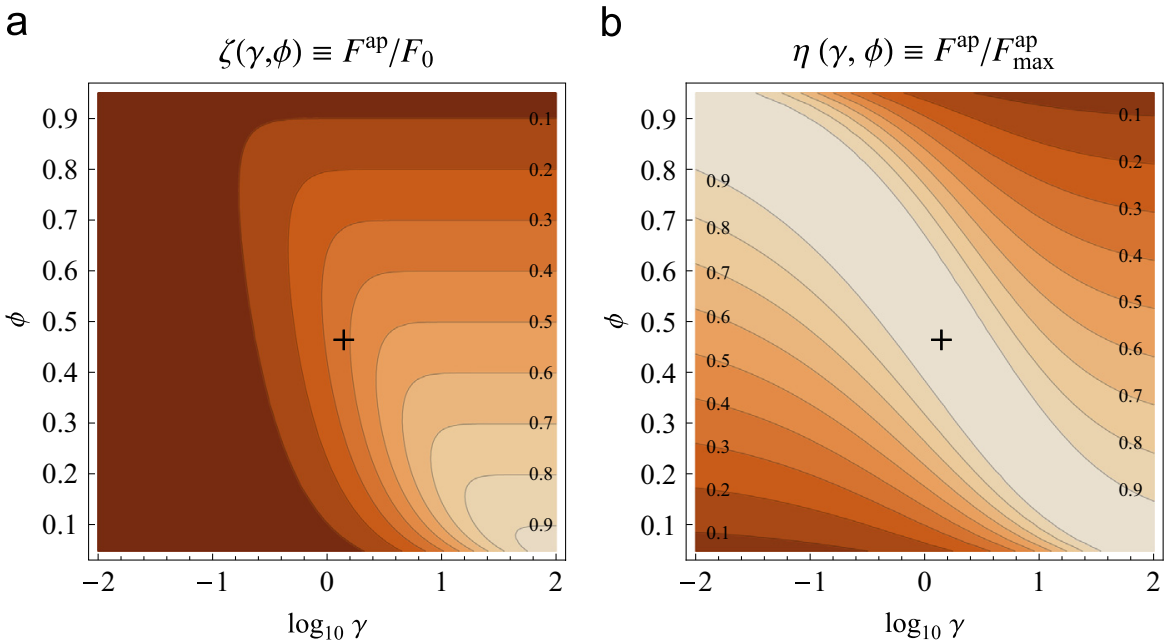

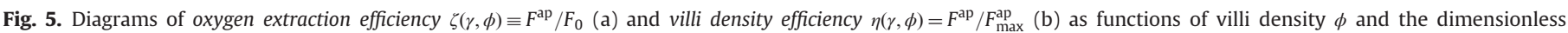

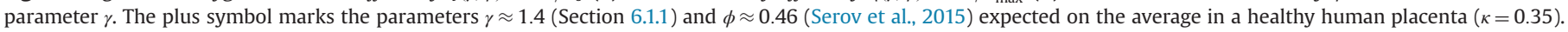


definition of $x$, one obtains $\phi_{\text {opt }}$ as a function of $\gamma$ :

$\phi_{\mathrm{opt}}(\gamma)=\frac{1}{1+\frac{\gamma}{g^{-1}(\gamma)}}$.

The function $\phi_{\text {opt }}(\gamma)$ (Fig. 6a) can be used to calculate an optimal villi density for a placental region if $\gamma$ is known for this region. Substitution of the last result into Eq. (25) gives the corresponding maximal uptake:

$\frac{F_{\max }^{\mathrm{ap}}(\gamma)}{F_{0}}=\gamma \frac{1-\exp \left(-g^{-1}(\gamma)\right)}{\gamma+g^{-1}(\gamma)}$.

From the asymptotic behavior of $g(x)$ at small and large $x$, we obtain the following asymptotic formulas for $\phi_{\mathrm{opt}}$ and $F_{\max }^{\mathrm{ap}} / F_{0}$ :

$\phi_{\text {opt }}(\gamma) \simeq\left\{\begin{array}{cl}\frac{1}{1+\sqrt{\gamma / 2}}, & \gamma \ll 1 \\ \ln (\gamma) / \gamma, & \gamma \gg 1\end{array} \quad \frac{F_{\max }^{\mathrm{ap}}(\gamma)}{F_{0}} \simeq \begin{cases}\frac{1-\mathrm{e}^{-\sqrt{2 \gamma}}}{1+\sqrt{2 / \gamma}}, & \gamma \ll 1 \\ \frac{1-1 / \gamma}{1+\ln (\gamma) / \gamma}, & \gamma \gg 1\end{cases}\right.$

Fig. 6 shows that these asymptotics accurately approximate $\phi_{\text {opt }}(\gamma)$ and $F_{\max }^{\mathrm{ap}}(\gamma) / F_{0}$ not only in the limits of $\gamma \ll 1$ and $\gamma \gg 1$, but for all $\gamma$. For instance, it can be calculated that if small- $\gamma$ asymptotic is used for $\gamma \leq 3$ and large- $\gamma$ asymptotic is used for $\gamma>3$, the maximal relative error of the second formula of Eq. (30) is less than $4 \%$. In other words, we obtained simple explicit approximations for the optimal villi density $\phi_{\text {opt }}$ and the normalized maximal oxygen uptake $F_{\max }^{\mathrm{ap}} / F_{0}$ as functions of a single parameter $\gamma$.

\subsection{Villi density efficiency}

Basing on the optimal villi density and the maximal uptake introduced in the previous section, one can define a quantitative measure of optimality of villi density in a given (not optimal) geometry.

If the given geometry is characterized by the parameters $(\gamma, \phi)$, its villi density efficiency can be defined as the ratio of oxygen uptake in this particular geometry to the maximal value, which can be obtained with the same $\gamma$ (Fig. 6b):

$\eta(\gamma, \phi) \equiv \frac{F^{\mathrm{ap}}(\gamma, \phi)}{F_{\max }^{\mathrm{ap}}(\gamma)}=\frac{(1-\phi)\left(1-\exp \left(-\gamma \frac{\phi}{1-\phi}\right)\right)}{\left(1-\phi_{\mathrm{opt}}(\gamma)\right)\left(1-\exp \left(-\gamma \frac{\phi_{\mathrm{opt}}(\gamma)}{1-\phi_{\mathrm{opt}}(\gamma)}\right)\right)}$.

Fig. 5b presents the villi density efficiency $\eta(\gamma, \phi)$ in a physiological range of $(\gamma, \phi)$ and at $L=L_{0}$.
Following the comment after Eq. (30), Eq. (31) can be rewritten as

$\eta(\gamma, \phi) \simeq \begin{cases}\frac{(1-\phi)\left(1-\exp \left(-\gamma \frac{\phi}{1-\phi}\right)\right)(1+\sqrt{2 / \gamma})}{1-\mathrm{e}^{-\sqrt{2 \gamma}}}, & \gamma \leq 3 \\ \frac{(1-\phi)\left(1-\exp \left(-\gamma \frac{\phi}{1-\phi}\right)\right)(1+\ln (\gamma) / \gamma)}{1-1 / \gamma}, & \gamma>3\end{cases}$

with a maximal relative error of $4 \%$. Note that the last equation does not require calculation of $\phi_{\mathrm{opt}}$ and is an explicit function of $\gamma$ and $\phi$.

Note finally that the optimality indicators $\zeta$ and $\eta$ play different roles. Oxygen extraction efficiency $\zeta$ (Fig. 5a) indicates the fraction of the maximal possible incoming oxygen flow $F_{0}$ that is absorbed by a given cross-sectional geometry. The higher is the value of $\zeta$, the higher is the absolute value of fetal oxygen uptake. At the same time, villi density efficiency $\eta$ (Fig. 5b) shows how far the villi density of a given cross-section is from its optimal value for a fixed $\gamma\left(r_{\mathrm{e}}\right)$. The higher is the value of $\eta$, the closer is fetal oxygen uptake to the maximal value for the given villi radius $r_{\mathrm{e}}$.

\section{Results}

Fig. 7 shows that fetal oxygen uptake predicted by the analytical equation (25) agrees well with numerically calculated results (Serov et al., 2015) in wide ranges of stream-tube lengths $(L)$ and villi densities $(\phi)$. Fig. 7a demonstrates the existence of maximal oxygen uptake corresponding to an optimal villi density for each stream-tube length. The value of $\kappa(L)$ was determined from Fig. 4b for each considered length $L$. These results were calculated for the same geometries as in our earlier numerical simulation (Fig. 8). We emphasize that numerical simulations with identical circular villi are shown only for the purpose of validation. The proposed analytical theory, which uses only villi density and the effective villi radius $\left(r_{\mathrm{e}}\right)$ as geometrical information, is applicable to villi of arbitrary shapes and sizes. The agreement of analytical curves with numerical points shows that for uniform villi distributions, knowing villi density and the effective villi radius is enough to predict the oxygen uptake.

Variations of the parameter $\gamma$ in analytical expressions for optimal villi density and maximal uptake (Eqs. (28) and (29)) can be interpreted in terms of changes of individual parameters of the model, other parameters being fixed. For example, optimal villi density and maximal uptake can be plotted as functions of MBF velocity (Fig. 9a and c) or stream-tube length (Fig. 9b and d). An agreement between the plotted curves and numerical results of Serov et al. (2015) can be observed. a

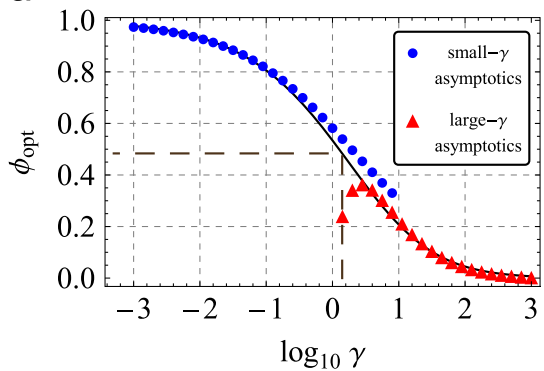

b

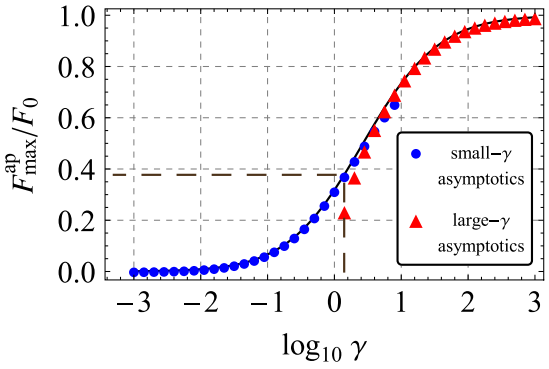

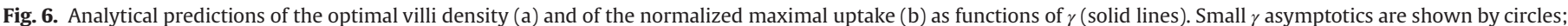
large $\gamma$ asymptotics are shown by triangles. Dashed lines marks $\gamma \approx 1.4$ observed in a healthy human placenta (see Section 6.1 .1 ). 
a

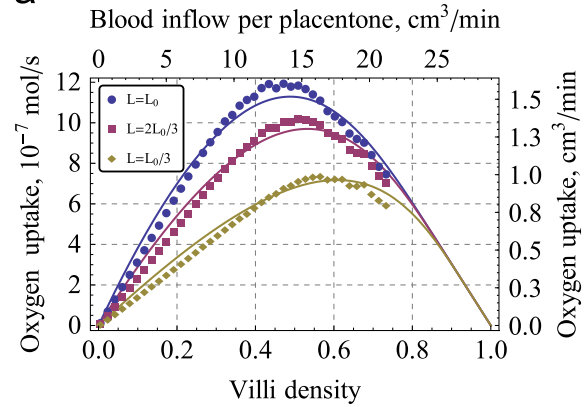

b

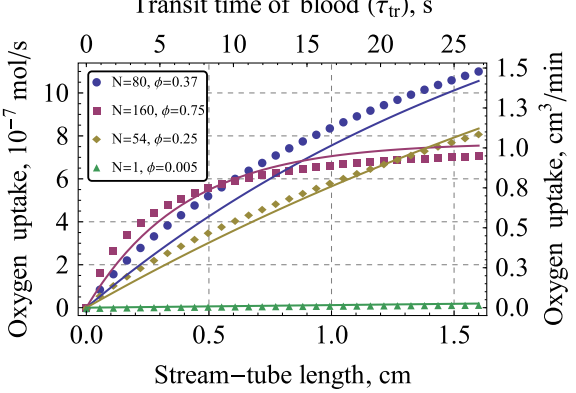

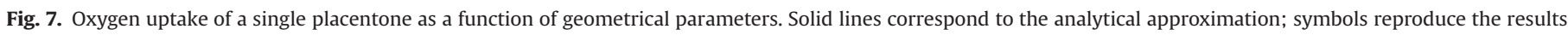

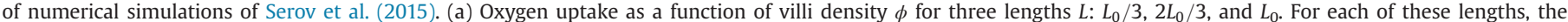

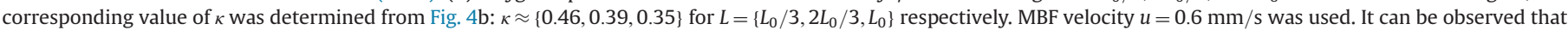

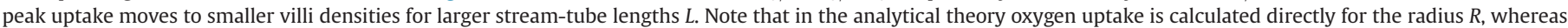

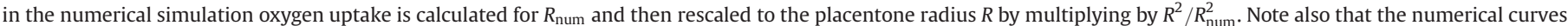

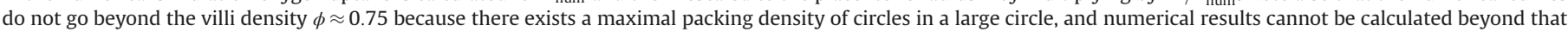

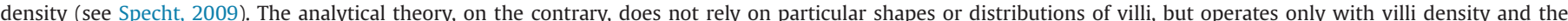

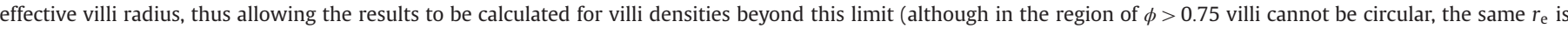

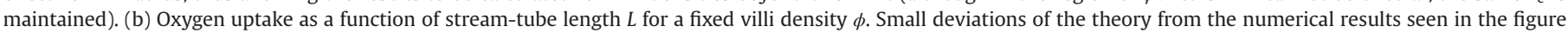
are explained by the fixed $\kappa=\kappa\left(L_{0}\right) \approx 0.35$ used for all lengths. Various symbols represent villi densities of Fig. 8.
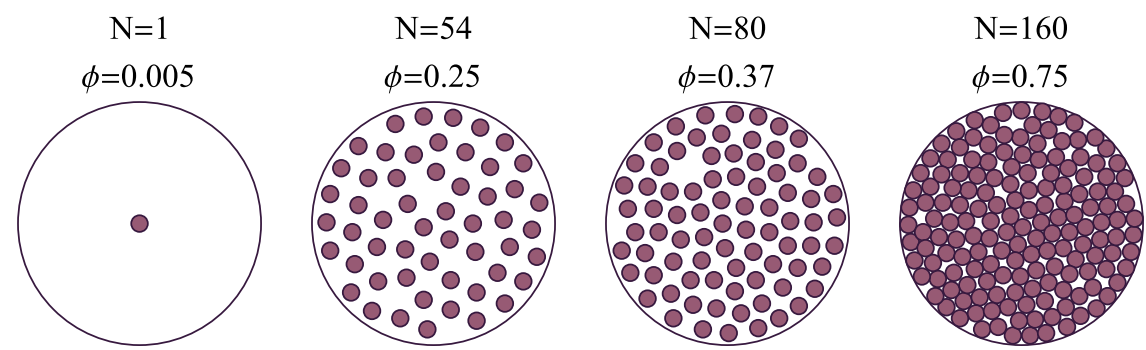

Fig. 8. Villi distributions for which oxygen uptake was calculated in Serov et al. (2015). Number of villi $(N)$ and the corresponding villi density $(\phi)$ are displayed above each case. The analytical theory was applied to the corresponding $\phi$ with $r_{\mathrm{e}}$ given in Table 1 . Maternal blood flows in the white space in the direction perpendicular to the crosssections.

a

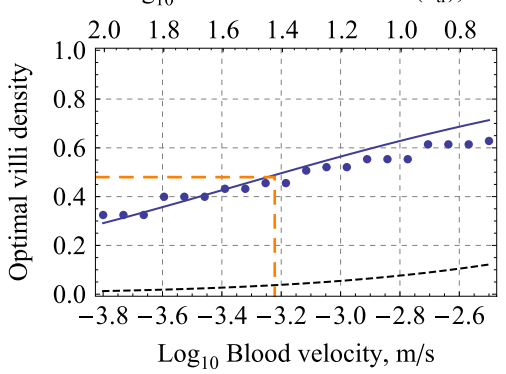

C

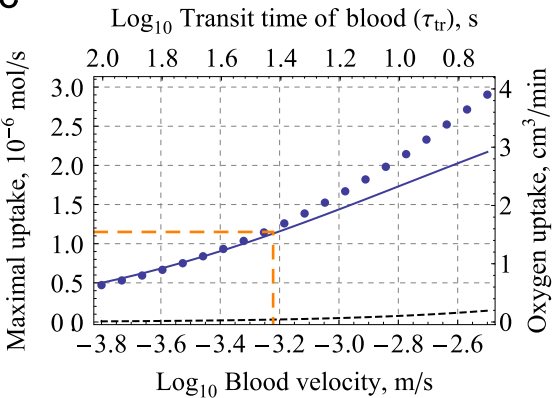

b

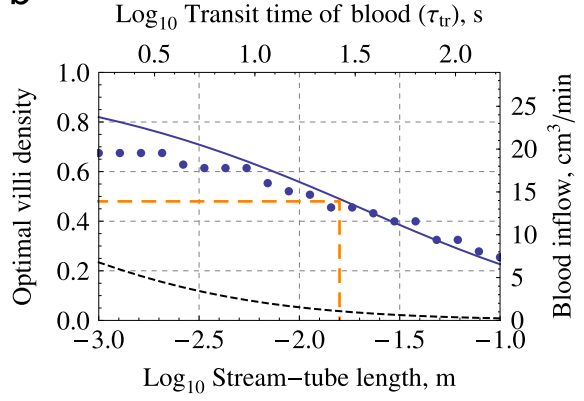

d

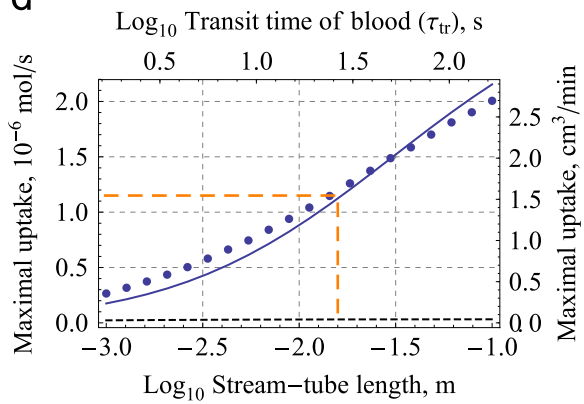

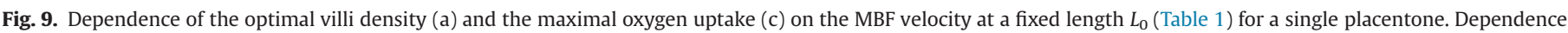

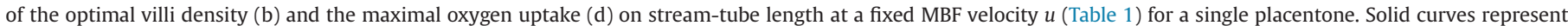

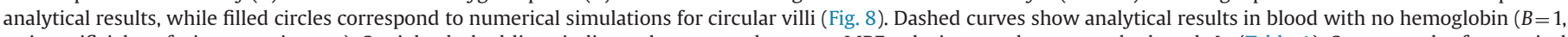

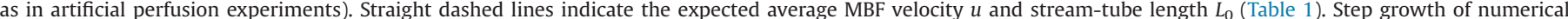

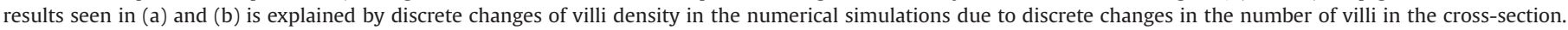


All four plots in Fig. 9 feature a dashed black curve representing a fictitious case of blood having no hemoglobin but transporting only oxygen dissolved in the blood plasma. Mathematically, this case is described by oxygen-hemoglobin dissociation parameter $B=1$, which is about 100 times smaller than that for blood with $\mathrm{Hb}$. As predicted by Eq. (28), the no-Hb curves for optimal villi density have the same shape but are shifted by two orders of magnitude as compared to those for normal blood.

\section{Discussion}

\subsection{Parameters $\gamma$ and $F_{0}$}

\subsubsection{Values}

Taking $\pi R^{2}$ as the total area of the cross-section, parameters from Table 1 and $\kappa\left(L_{0}\right) \approx 0.35$ for the average stream-tube length $L_{0} \approx 1.6 \mathrm{~cm}$ (Fig. 4b), from Eq. (26) one can estimate the values of $\gamma$ and $F_{0}$ which characterize a "healthy" regime of our placenta model: $\gamma \approx 1.4, F_{0} \approx 3 \cdot 10^{-6} \mathrm{~mol} / \mathrm{s}$. The obtained average value of $\gamma$ together with the average villi density $\phi \approx 0.46$ (Serov et al., 2015) are marked by crosses in the diagrams in Fig. 5. One can see that the theory predicts that an average placenta extracts around $35 \%$ of the maximal possible incoming oxygen flow $F_{0}$ (Fig. 5a), and that this value is close to the maximal one for the given effective villi radius (Fig. $5 \mathrm{~b}$ ). Although $35 \%$ seems to be a low value, note that oxygen extraction efficiency of $100 \%$ is never achievable since in the presence of villi only a part of the flow unobstructed by villi $\left(F_{0}\right)$ can be transferred to the fetus.

To have predictive power, $\gamma$ and $\phi$ need to be measured for different healthy as well as pathological placentas over the whole exchange region. Such measurements require development of image analysis techniques, which could automatically determine these characteristics for histological placental slides. Such measurements have not yet been performed and present an important perspective to this study. At the same time, because of the lack of experimental information about several other parameters (namely, $u, w$, and $L$ ) in each studied placenta, correlations of changes of $\gamma$ and $\phi$ with changes of fetal development characteristics (such as birth-weight, placenta weight or their ratio) are expected to be of more practical use than absolute values of $\gamma$ and $\phi$. Note finally that the optimal geometry and maximal uptake may change for non-slip boundary conditions; further studies are required to clarify this point (for discussion see Serov et al., 2015).

\subsubsection{The optimal villi density: the parameter}

The two parameters $\gamma$ and $F_{0}$ play different roles. According to Eq. (27), $\gamma$ alone determines the optimal villi density, while $F_{0}$ together with $\gamma$ determines the maximal oxygen uptake (Eq. (29)). A clear physical interpretation of $\gamma$ can be obtained by rewriting (26) as

$\gamma=\frac{L / u}{B r_{\mathrm{e}} /(2 w \kappa)}=\frac{\tau_{\text {tr }}}{\tau_{\mathrm{e}}}$

where $\tau_{\text {tr }} \equiv L / u$ is the transit time of maternal blood through the placenta (while it flows along a stream tube of length $L$ with an average velocity $u$ ) and $\tau_{\mathrm{e}} \equiv B r_{\mathrm{e}} /(2 w \kappa)$ is oxygen extraction time of a placental cross-section. As a consequence, $\gamma$ can be understood as a quantitative measure of balance between two oxygen transport mechanisms: the longitudinal convective flow and the transverse diffusion. In other words, $\gamma$ describes the level of adaptation of the geometry of the cross-section and uptake parameters to the incoming MBF. Large values of $\gamma(\gamma \gg 1)$ mean that oxygen is quickly transferred to the fetal circulation at the beginning of the stream-tube and is rapidly depleted, so that poor in oxygen maternal blood flows through the remaining part.
Thus, this remaining part does not function efficiently. Small values of $\gamma(\gamma \ll 1)$ mean that maternal blood passes too quickly through the placenta as compared to the oxygen extraction time, so that a considerable part of the incoming oxygen flow may not be transferred. One can then speculate that transport of oxygen is the most efficient in the placentas, for which $\gamma$ is of the order of 1 . $\gamma \approx 1.4$ calculated from the model parameters suggests that a healthy placenta may indeed function optimally.

\subsection{The analytical theory}

The advantages of the analytical solution over the numerical one are numerous:

1. Oxygen uptake can be estimated for a histological cross-section of arbitrary geometry.

2. The villi density $\phi$ and the effective villi radius $r_{\mathrm{e}}$ are shown to be the only geometrical parameters necessary to predict oxygen uptake of a rather uniform villi distribution in a placental cross-section (see Figs. 7 and 9). These two parameters allow for a simple application of the theory to distributions of villi of arbitrary shapes. The validity of the theory in the case of strongly non-uniform villi distributions remains to be investigated.

Finer details of villi distributions which produce differences between numerical and analytical results in Figs. 7 and 9, are "stored" in the coefficient $\kappa$. This coefficient encompasses not only the details of villi distributions, but also determines the strength of their influence on oxygen uptake at a given length $L$. In other words, it quantitatively describes the fact that in each geometry, different regions of the IVS are not equivalent due to random distribution of villi, and that with length $L$, oxygen in some regions is exhausted faster than in other regions. However, the dependence of $\kappa$ and $\alpha$ on $L$ is rather weak as can be seen in Fig. 7b, in which $F^{\mathrm{ap}}(L)$ is plotted for all lengths with the same $\kappa=\kappa\left(L_{0}\right) \approx 0.35$. In the first approximation, $\kappa$ can hence be considered independent of $L$.

3. The efficiency of oxygen transport in a given placental crosssection can be estimated by means of oxygen extraction efficiency $\zeta$ and villi density efficiency $\eta$ plotted in Fig. 5. For these two quantities, simple analytical formulas and diagrams are provided, which allow for comparison of different placentas or placental regions once the parameters $\phi$ and $\gamma$ are calculated for them. To have predictive power, the efficiency estimations provided by the model need to be studied for correlations with independent indicators of placental exchange efficiency, such as placenta shape, placenta weight, placenta-fetus birth-weight ratio (Misra et al., 2010; Hutcheon et al., 2012) or pulsatility indices determined by Doppler velocimetry in the umbilical cord or maternal vessels (Todros et al., 1999; Madazli et al., 2003), which were demonstrated to vary between normal and pre-eclamptic pregnancies or pregnancies complicated by fetal growth restriction.

4. The analytical theory suggests that oxygen uptake in the human placenta is rather robust to changes of villi density. Indeed, the diagram in Fig. 5a shows that placental villi density can vary by about $10 \%$ around the optimal value with the villi density efficiency $\eta$ staying in the $90-100 \%$ interval. Far from the optimal villi density, $\eta$ tends to decrease faster.

5. One can to analyze the consequences of neglecting oxygenhemoglobin reaction on the predictions of oxygen uptake and the optimal villi density of a placental region. Moreover, the theory gives a method of recalculation of the results obtained for no-Hb blood in artificial placenta perfusion experiments into those for normal blood. Imagine that at the end of an artificial perfusion experiment with no-Hb blood, one obtains 
a

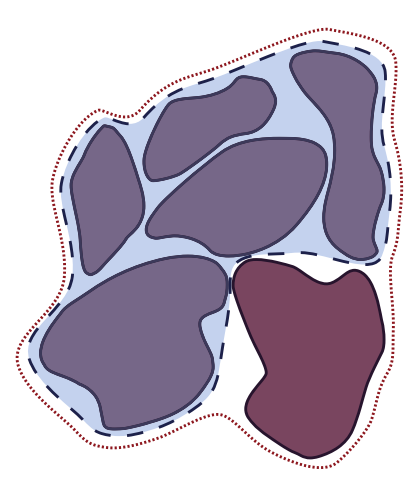

b

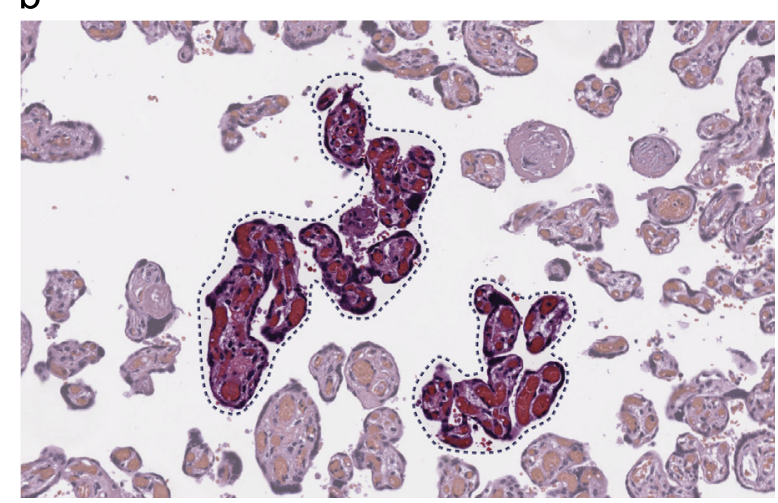

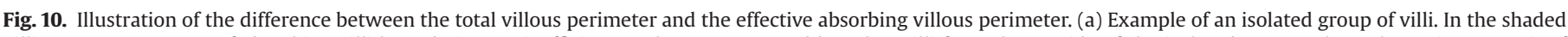

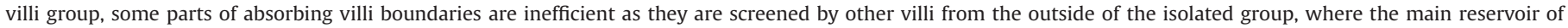

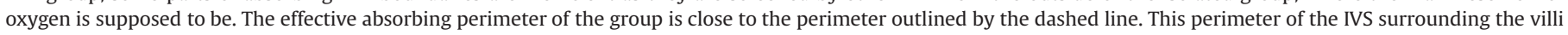

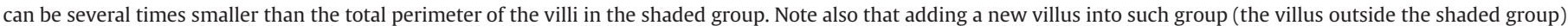

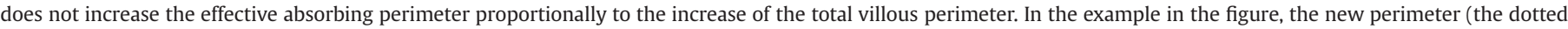

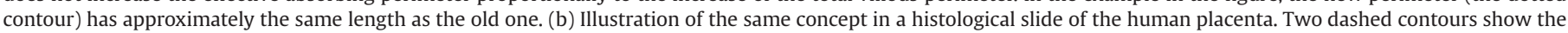

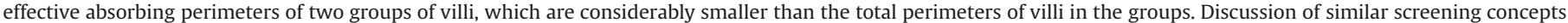
can be found in Sapoval et al. (2002), Felici et al. (2005), and Gill et al. (2011).

the total oxygen inflow $\tilde{F}_{\text {in }}$ into the placenta, fetal oxygen uptake $\tilde{F}$ and the average villi density $\phi$ from histomorphometry of the same placenta (note that $\tilde{F}_{\text {in }}$ and $\tilde{F}$ differ from $F_{\text {in }}$ and $F$ which would have been obtained for normal blood). From these data one can calculate $\tilde{F}_{0}=\tilde{F}_{\text {in }} /(1-\phi)$ (see the discussion of Eq. (26)) and then $\tilde{\gamma}$ as a root of Eq. (25) (with $F^{\text {ap }}$ replaced by $\tilde{F})$. These values can be recalculated for blood containing $\mathrm{Hb}$ : $\gamma=\tilde{\gamma} / B$ and $F_{0}=\tilde{F}_{0} B$, where $B \approx 94$ (Table 1 ), and can be substituted into Eq. (25) to give oxygen uptake $F$ in the same placenta for blood containing $\mathrm{Hb}$. One can see that oxygen uptake in a no-Hb perfusion experiment gives on the average a hundred-times underestimation of the real uptake. Finally, the values of $\gamma$ and $\phi$ for the given placenta can be compared with the diagram in Fig. 5b to determine how far the geometry of the region is from the optimal one. Note that this recalculation introduces a small error as in no-Hb case the diffusive part of the total flow, which is omitted in Eq. (17), becomes important.

6. The computation time is reduced since calculations of eigenfunctions and eigenvalues of the diffusion equation are not required. Note that due to long computation time, numerical simulations of Serov et al. (2015) had to be performed on a smaller placentone radius $R_{\text {num }}$ and then rescaled to the radius $R$ by multiplying oxygen uptake by $R^{2} / R_{\text {num }}^{2}$. This constraint does not apply to the analytical theory. In particular, good agreement between both approaches justifies the rescaling of results performed in the numerical calculations.

Note finally that the derivation of Eq. (26) implies that, strictly speaking, $P$ is not the total perimeter of the villi, but the effective absorbing perimeter of the villi (i.e. only its part that is directly in contact with the IVS). In the case of well-separated villi, there is no difference between the two definitions. However, it is not always the case in placental cross-sections. For instance, in Fig. 10b one can see several isolated groups of villi, inside which villi lie so close to each other, that there is virtually no IVS left between them. Parts of the villous boundary which are not in contact with large parts of the IVS are then screened from participating in oxygen uptake, and hence should not be accounted for in the effective absorbing perimeter of the villi. This remark can be understood by considering the fact that oxygen diffuses in the IVS, and only parts of the villous boundary that are in contact with the IVS will participate in the uptake. In the case of well separated singular villi, the entire perimeter is absorbing. A schematic description of this situation is shown in Fig. 10a. Note finally that in our model the screening effect is implicitly taken into account by the diffusion equation (10).

\section{Conclusions}

In the present work, an analytical solution to the diffusionconvection equation governing oxygen transport in the human placenta has been developed. Oxygen uptake was calculated for an arbitrary cross-sectional geometry of the stream tubes of maternal blood. It was shown that for a rather uniform spatial distribution of villi in a placental cross-section, only two geometrical characteristics, villi density $\phi$ and the effective villi radius $r_{\mathrm{e}}$, are needed to predict fetal oxygen uptake.

It was also demonstrated that all the parameters of the model do not influence oxygen uptake independently, but instead form two combinations: (i) the maximal oxygen inflow of one placentone $F_{0}$ and (ii) the ratio $\gamma$ of the transit time of maternal blood through the IVS and the oxygen extraction time. These two parameters together with villi density determine oxygen uptake. Analytical formulas and diagrams were obtained which allow for oxygen uptake calculation and quantitative estimation of the efficiency of oxygen transport of a given placental region based on measurements of $\phi$ and $r_{\mathrm{e}}$.

Finally, a fictitious case of blood containing no hemoglobin was analyzed to study oxygen transport in artificial placenta perfusion experiments. It was demonstrated that artificial perfusion experiments with no hemoglobin tend to give a two-orders-of-magnitude underestimation of the in vivo oxygen uptake. A method of recalculation of the results of artificial perfusion experiments to account for oxygen-hemoglobin dissociation was proposed.

Once combined with image analysis techniques, the proposed analytical theory can be the mathematical ground for a future tool of fast diagnostics of placenta efficiency based on histological placental slides.

\section{Acknowledgments}

The authors thank Dr. Paul Brownbill for fruitful discussions.

This study was funded by the International Relations Department of Ecole Polytechnique as a part of the Ph.D. project of A.S. 
Serov, by Placental Analytics LLC, NY, by SAMOVAR project of the Agence Nationale de la Recherche no 2010-BLAN-1119-05 and by Agence Nationale de la Recherche project ANR-13-JSV5-0006-01.

\section{References}

Aifantis, E.C., 1978. Towards a rational modeling for the human placenta. Math Biosci. 40 (3-4), 281-301. http://dx.doi.org/10.1016/0025-5564(78)90090-1.

Arfken, G.B., Weber, H.J., Harris, F.E., 2005. Mathematical Methods for Physicists, 6th edition Academic Press, Orlando, FL.

Bacon, B.J., Gilbert, R.D., Longo, L.D., 1986. Regional anatomy of the term human placenta. Placenta 7 (3), 233-241. http://dx.doi.org/10.1016/S0143-4004(86) 80161-8, URL: 〈http://www.ncbi.nlm.nih.gov/pubmed/3737577〉.

Bartels, H., Moll, W., Metcalfe, J., 1962. Physiology of gas exchange in the human placenta. Am. J. Obstet. Gynecol. 84, 1714-1730, URL: 〈http://www.ncbi.nlm.nih. gov/pubmed/13969669).

Battaglia, F.C., Meschia, G., 1986. An Introduction to Fetal Physiology. Academic Press, Inc., London.

Benirschke, K., Kaufmann, P., Baergen, R.N., 2006. Pathology of the Human Placenta, 5 th edition Springer, NY.

Burchell, R.C., 1967. Arterial blood flow into the human intervillous space. Am. J. Obstet. Gynecol. 98 (3), 303-311, URL: 〈http://www.ncbi.nlm.nih.gov/ pubmed/5621448>.

Challier, J.-C., Uzan, S., 2003. Le placenta humain et ses pathologies: l'oxygène en question. Med. Sci. (Paris) 19 (11), 1111-1120. http://dx.doi.org/10.1051/medsci/ 200319111111, URL: 〈http://www.ncbi.nlm.nih.gov/pubmed/14648482〉.

Chernyavsky, I.L., Jensen, O.E., Leach, L., 2010. A mathematical model of intervillous blood flow in the human placentone. Placenta 31 (1), 44-52. http://dx.doi.org/ 10.1016/j.placenta.2009.11.003, URL: 〈http://www.ncbi.nlm.nih.gov/pubmed/ 19945160).

Erian, F.F., Corrsin, S., Davis, S.H., 1977. Maternal, placental blood flow: a model with velocity-dependent permeability. J. Biomech. 10 (11/12), 807-814. http: //dx.doi.org/10.1016/0021-9290(77)90095-1, URL: 〈http://www.ncbi.nlm.nih. gov/pubmed/606727 $\rangle$.

Faber, J.J., 1969. Application of the theory of heat exchangers to the transfer of inert materials in placentas. Circ. Res. 24 (2), 221-234. http://dx.doi.org/10.1161/01. RES.24.2.221, URL: 〈http://www.ncbi.nlm.nih.gov/pubmed/5764583〉.

Felici, M., Filoche, M., Straus, C., Similowski, T., Sapoval, B., 2005. Diffusional screening in real 3D human acini-a theoretical study. Respir. Physiol. Neurobiol. 145 (2-3), 279-293. http://dx.doi.org/10.1016/j.resp.2004.10.012, URL: 〈http://www.ncbi.nlm.nih.gov/pubmed/15705542〉.

Foucquier, A., Filoche, M., Moreira, A.A., Andrade, J.S., Arbia, G., Sapoval, B., 2013. A first principles calculation of the oxygen uptake in the human pulmonary acinus at maximal exercise. Respir. Physiol. Neurobiol. 185 (3), 625-638. http: //dx.doi.org/10.1016/j.resp.2012.10.013, URL: 〈http://www.ncbi.nlm.nih.gov/ pubmed/23201099〉.

Gill, J.S., Salafia, C.M., Grebenkov, D.S., Vvedensky, D.D., 2011. Modeling oxygen transport in human placental terminal villi. J. Theor. Biol. 291, 33-41. http://dx. doi.org/10.1016/j.jtbi.2011.09.008, URL: 〈http://www.ncbi.nlm.nih.gov/pubmed/ 21959313>.

Gray, H., 1918. Anatomy of the Human Body, 20th edition Lea \& Febiger, Philadelphia.

Groome, L.J., 1991. A theoretical analysis of the effect of placental metabolism on fetal oxygenation under conditions of limited oxygen availability. Biosystems 26 (1), 45-56. http://dx.doi.org/10.1016/0303-2647(91)90036-K, URL: 〈http:/| www.ncbi.nlm.nih.gov/pubmed/1760534〉.

Guilbeau, E.J., Reneau, D.D., Knisely, M.H., 1970. A distributed parameter mathematical analysis of oxygen exchange from maternal to fetal blood in the human placenta. In: Hershey, D. (Ed.), Proceedings of the International Symposium on Blood Oxygenation, held at the University of Cincinnati, December 1-3, 1969. Plenum, New York, pp. 218-221.

Hill, E.P., Power, G.G., Longo, L.D., 1972. A mathematical model of placental 02 transfer with consideration of hemoglobin reaction rates. Am. J. Physiol. 222 (3), 721-729, URL: 〈http://www.ncbi.nlm.nih.gov/pubmed/5022686〉.

Hill, E.P., Power, G.G., Longo, L.D., 1973. A mathematical model of carbon dioxide transfer in the placenta and its interaction with oxygen. Am. J. Physiol. 224 (2), 283-299, URL: 〈http://www.ncbi.nlm.nih.gov/pubmed/4686484〉.

Hutcheon, J.A., McNamara, H., Platt, R.W., Benjamin, A., Kramer, M.S., 2012. Placental weight for gestational age and adverse perinatal outcomes. Obstet. Gynecol. 119 (6), 1251-1258. http://dx.doi.org/10.1097/AOG.0b013e318253d3df, URL: 〈http:// www.ncbi.nlm.nih.gov/pubmed/22617591〉.

Jauniaux, E., Watson, A.L., Hempstock, J., Bao, Y.-P., Skepper, J.N., Burton, G.J., 2000. Onset of maternal arterial blood flow and placental oxidative stress. Am. J. Pathol. 157 (6), 2111-2122. http://dx.doi.org/10.1016/S0002-9440(10)64849-3, URL: 〈http://www.ncbi.nlm.nih.gov/pmc/articles/PMC1885754〉.

Kirschbaum, T.H., Shapiro, N.Z., 1969. A mathematical model of placental oxygen transfer. J. Theor. Biol. 25 (3), 380-402. http://dx.doi.org/10.1016/S0022-5193 (69)80028-7, URL: 〈http://www.ncbi.nlm.nih.gov/pubmed/5394213〉.
Lardner, T.J., 1975. A model for placental oxygen exchange. J. Biomech. 8 (2), 131-134. http://dx.doi.org/10.1016/0021-9290(75)90093-7, URL: 〈http://www. ncbi.nlm.nih.gov/pubmed/1150679).

Law, R., Bukwirwa, H., 1999. The physiology of oxygen delivery. Updat. Anaesth. 10 8-14. http://dx.doi.org/10.1111/j.1741-6892.2004.00434.x, URL:〈http://www. ncbi.nlm.nih.gov/pubmed/16214521>.

Longo, L.D., Hill, E.P., Power, G.G., 1972a. Theoretical analysis of factors affecting placental 02 transfer. Am. J. Physiol. 222 (3), 730-739, URL: 〈http://www.ncbi nlm.nih.gov/pubmed/5022687

Longo, L.D., Hill, E.P., Power, G.G., 1972b. Factors affecting placental oxygen transfer. In: Longo, L.D., Bartels, H. (Eds.), Respiratory Gas Exchange and Blood Flow in the Placenta. National Institute of Child Health and Human Development, Bethesda, MD, pp. 345-393.

Madazli, R., Somunkiran, A., Calay, Z., Ilvan, S., Aksu, M.F., 2003. Histomorphology of the placenta and the placental bed of growth restricted foetuses and correlation with the Doppler velocimetries of the uterine and umbilical arteries. Placenta 24 (5), 510-516, URL: 〈http://www.ncbi.nlm.nih.gov/pubmed/12744927〉.

Mayhew, T.M., Jairam, I.C., 2000. Stereological comparison of 3D spatial relationships involving villi and intervillous pores in human placentas from control and diabetic pregnancies. J. Anat. 197 (Pt 2), 263-274. http://dx.doi.org/10.1046 j.1469-7580.2000.19720263.x, URL: 〈http://www.ncbi.nlm.nih.gov/pubmed/ $11005718\rangle$.

Mayhew, T.M., Joy, C.F., Haas, J.D., 1984. Structure-function correlation in the human placenta: the morphometric diffusing capacity for oxygen at full term. J. Anat 139 (Pt 4), 691-708, URL: 〈http://www.ncbi.nlm.nih.gov/pubmed/6526720〉.

Mayhew, T.M., Jackson, M.R., Haas, J.D., 1986. Microscopical morphology of the human placenta and its effects on oxygen diffusion: a morphometric model. Placenta 7 (2), 121-131. http://dx.doi.org/10.1016/S0143-4004(86)80003-0, URL: 〈http://www.ncbi.nlm.nih.gov/pubmed/3725744〉.

Metcalfe, J., Moll, W., Bartels, H., 1964. Gas exchange across the placenta. Fed. Proc. 23, 774-780, URL: 〈http://www.ncbi.nlm.nih.gov/pubmed/14195461〉.

Misra, D.P., Salafia, C.M., Charles, A.K., Miller, R.K., 2010. Birth weights smaller or larger than the placenta predict BMI and blood pressure at age 7 years. J. Dev. Orig. Health Dis. 1 (02), 123. http://dx.doi.org/10.1017/S2040174410000139, URL: 〈http://www.journals.cambridge.org/abstract_S2040174410000139〉.

Moll, W., 1972. Gas exchange in concurrent. In: Longo, L.D., Bartels, H. (Eds.) Respiratory Gas Exchange and Blood Flow in the Placenta. National Institute of Child Health and Human Development, Bethesda, MD, pp. 281-296.

Power, G.G., Hill, E.P., Longo, L.D., 1972a. Analysis of uneven distribution of diffusing capacity and blood flow in the placenta. Am. J. Physiol. 222 (3), 740-746, URL: 〈http://www.ncbi.nlm.nih.gov/pubmed/5022688〉.

Power, G.G., Hill, E.P., Longo, L.D., 1972b. A mathematical model of carbon dioxide transfer in the placenta. In: Longo, L.D., Bartels, H. (Eds.), Respiratory Gas Exchange and Blood Flow in the Placenta. National Institute of Child Health and Human Development, Bethesda, MD, pp. 395-418.

Rodesch, F., Simon, P., Donner, C., Jauniaux, E., 1992. Oxygen measurements in endometrial and trophoblastic tissues during early pregnancy. Obstet. Gynecol. 80 (2), 283-285, URL: 〈http://www.ncbi.nlm.nih.gov/pubmed/1635745〉.

Sapoval, B., Filoche, M., Weibel, E.R., 2002. Smaller is better-but not too small: a physical scale for the design of the mammalian pulmonary acinus. Proc. Natl. Acad. Sci. USA 99 (16), 10411-10416. http://dx.doi.org/10.1073/pnas.122352499, URL: 〈http://www.ncbi.nlm.nih.gov/pubmed/12136124〉.

Schröder, H., 1982. Structural and functional organization of the placenta from the physiological point of view. In: Kaufmann, P., King, B.F. (Eds.) Structural and Functional Organization of the Placenta. Karger, Basel, pp. 4-12, URL: 〈http://www.ncbi.nlm.nih.gov/pubmed/7126150〉.

Serov, A.S., Salafia, C.M., Brownbill, P., Grebenkov, D.S., Filoche, M., 2015. Optima villi density for maximal oxygen uptake in the human placenta. J. Theor. Biol. 364, 383-396. http://dx.doi.org/10.1016/j.jtbi.2014.09.022, URL: 〈http://www. ncbi.nlm.nih.gov/pubmed/25261730>.

Severinghaus, J.W., 1979. Simple, accurate equations for human blood 02 dissociation computations. J. Appl. Physiol. 46 (3), 599-602, URL: 〈http://www.ncbi. nlm.nih.gov/pubmed/35496>.

Shapiro, N.Z., Kirschbaum, T.H., Assali, N.S., 1967. Mental exercises in placenta transfer. Am. J. Obstet. Gynecol. 97 (1), 130-137, URL: 〈http://www.ncbi.nlm. nih.gov/pubmed/6068096〉.

Specht, E., Packomania web-site (〈http://www.packomania.com〉), 2009. (accessed 31.12.2014)

Todros, T., Sciarrone, A., Piccoli, E., Guiot, C., Kaufmann, P., Kingdom, J., 1999 Umbilical Doppler waveforms and placental villous angiogenesis in pregnancies complicated by fetal growth restriction. Obstet. Gynecol. 93 (4), 499-503. http: //dx.doi.org/10.1016/S0029-7844(98)00440-2, URL:〈http://www.ncbi.nlm.nih. gov/pubmed/10214822>

Wilbur, W.J., Power, G.G., Longo, L.D., 1978. Water exchange in the placenta: a mathematical model. Am. J. Physiol. 235 (3), R181-R189, URL: 〈http://www. ncbi.nlm.nih.gov/pubmed/567941).

Yamaguchi, K., Nguyen-Phu, D., Scheid, P., Piiper, J., 1985. Kinetics of O2 uptake and release by human erythrocytes studied by a stopped-flow technique. J. Appl. Physiol. 58 (4), 1215-1224, URL: 〈http://www.ncbi.nlm.nih.gov/pubmed/ $3988677\rangle$. 